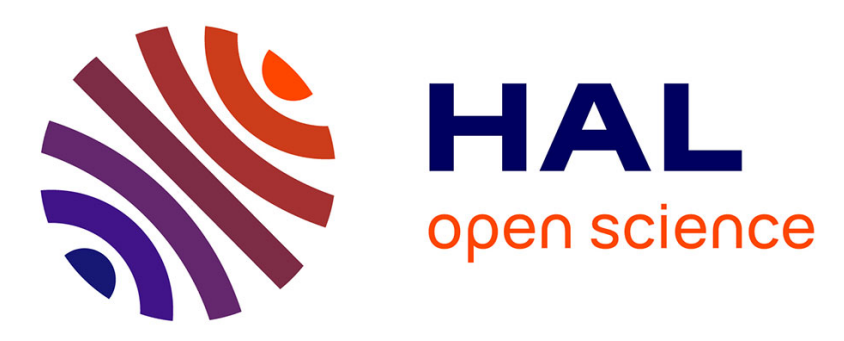

\title{
A moisture diffusion coupled model for composite materials
}

\author{
Djelloul Gueribiz, Frédéric Jacquemin, Sylvain Fréour
}

\section{To cite this version:}

Djelloul Gueribiz, Frédéric Jacquemin, Sylvain Fréour. A moisture diffusion coupled model for composite materials. European Journal of Mechanics - A/Solids, 2013, 42, pp.81-89. 10.1016/j.euromechsol.2013.04.008 . hal-01004849

\section{HAL Id: hal-01004849 \\ https://hal.science/hal-01004849}

Submitted on 12 Dec 2018

HAL is a multi-disciplinary open access archive for the deposit and dissemination of scientific research documents, whether they are published or not. The documents may come from teaching and research institutions in France or abroad, or from public or private research centers.
L'archive ouverte pluridisciplinaire HAL, est destinée au dépôt et à la diffusion de documents scientifiques de niveau recherche, publiés ou non, émanant des établissements d'enseignement et de recherche français ou étrangers, des laboratoires publics ou privés. 


\title{
A moisture diffusion coupled model for composite materials
}

\author{
D. Gueribiz ${ }^{\mathrm{a}}$, F. Jacquemin ${ }^{\mathrm{b}, *}$, S. Fréour ${ }^{\mathrm{b}}$ \\ a Laboratoire de Génie des Procédés, Université de Laghouat, Algeria \\ ${ }^{\mathrm{b}}$ Institut de Recherche en Génie Civil et Mécanique (UMR CNRS 6183), LUNAM Université, Université de Nantes, Ecole Centrale de Nantes, 37, \\ Bd de l'Université, BP 406, 44602 Saint-Nazaire, France
}

\begin{abstract}
In this study, a coupled diffusive behaviour model for a polymer matrix composite is proposed. Within this model, the diffusion of moisture depends upon the stress state experienced by the material. The model constitutive equations are based on a thermo-dynamical approach. The model is, firstly, established for a homogeneous isotropic matrix. It is, thereafter, extended to impermeable unidirectional composites. The stress-diffusion relation has been treated through a two steps procedure. In a first step, only the coupling between the maximum moisture absorption capacity and the stresses is assumed to occur. This implicitly involves that the diffusivity remains independent upon the mechanical states. In a second step, both the diffusivities and maximum moisture absorption capacity were expressed as functions of the mechanical states.
\end{abstract}

\section{Introduction}

The moisture diffusion problem in polymer matrix composites is often investigated through uncoupled models (Crank, 1975; Loos and Springer, 1979, 1981; Shen and Springer, 1977; Tsai and Hahn, 1980; Jacquemin and Vautrin, 2002). Fick's model in particular, although easy to implement (numerically) has repeatedly shown its limits when confronted to experimental data. This statement especially applies to the context of simulating long-term diffusive behaviour experienced by composite materials (Weitsman et al., 1987; Whitney and Browning, 1978; Jiming and James, 1995). Discrepancies between Fick's law predictions and the practically measured quantities are sometimes described as anomalies of diffusion (Perreux et al., 2002; Avena and Bunsell, 1986). Some of the so-called anomalous diffusive behaviours can be attributed to, and explained by, a hygro-mechanical coupling, featuring relations between the diffusion process and the internal mechanical states experienced by the material (Henson and Weitsman, 1986; Weitsman, 1990; Lee and Peppas, 1993).

In this study, a more realistic modelling of composites diffusive behaviour taking into account a hygro-mechanical coupling is investigated. Within this approach, the effective diffusivity of a composite ply is related to the mechanical states experienced by its very constituents, i.e. the organic matrix and the reinforcing fibres. Such a hygro-mechanical coupling affects both the coefficients of

\footnotetext{
* Corresponding author.

E-mail address: frederic.jacquemin@univ-nantes.fr (F. Jacquemin).
}

diffusion and the maximum moisture absorption capacity. The principle of the model is founded on Larché and Cahn theory (1973, 1978, 1982), developed for investigating diffusion of gases in metals. This theory has already been extended to pure polymers by Neogi and Kim (1984), Neogi et al. (1986), Carbonell and Sarti (1990), Derrien and Gilormini (2006, 2007). The specific case of heterogeneous composites was investigated by Durier et al. (2006) and (Derrien and Gilormini, 2007). Recently, another attempt in this trend has been proposed by Youssef et al. (2009a,b), for a laminate. In the present work, we attempt to study the diffusive behaviour of composite with polymer matrix taking into account the stress-diffusion interaction owing to aso-called diffusive coupled model. A coupled model description for a homogeneous isotropic matrix based upon thermodynamics is firstly presented. The model is thereafter extended to deal with impermeable unidirectional fibre composites. As part of the developed approach, the stress-diffusion interaction will be treated through a two steps procedure. In a first step, only the coupling between the maximum moisture absorption capacity and the stresses is assumed to occur. This implicitly involves that the diffusivity remains independent upon the mechanical states. In a second step, both the diffusivities and maximum moisture absorption capacity were expressed as functions of the mechanical states.

\section{Coupled model description}

The diffusion flux is usually modelled using Fick's law, which has the advantage of being simple. Unfortunately, for materials such as polymers and composites, which are very sensitive to moisture and 
can undergo swelling, transport phenomena are more complex. In fact, the diffusivity, assumed constant (for a given isotherm) in Fick's law, may in some cases, depend upon the moisture content. Such an occurrence induces non-linearities, the appropriate representation of which requires more advanced models.

The coupling between diffusion and stresses is often established accordingly to Larché and Cahn (1973) theory. Larché and Cahn studied the moisture concentration and internal stresses influence on the local diffusion flow. In order to theoretically account for these effects, the authors considered the moisture flux as independent upon both the stresses and concentration gradients. On the opposite, the traditional Fickian model neglects the effects, induced by the mechanical states on the diffusion process. As a consequence, Larché and Cahn theory constitutes an improvement over Fick's historical proposal. Nevertheless, it should be stressed, that Larché and Cahn model was developed for dealing with the issue of hydrogen diffusion in metals. Thus, this model applies well to many materials, provided that they exhibit a linear isotropic elastic behaviour. The state variables chosen for building this theoretical approach are the strains, the entropy, and the chemical species concentrations.

In the context of diffusion through heterogeneous solids, a potential $\mu$, constant at equilibrium, can be defined. This potential is the chemical potential, in the cases that fluids diffusing through solids are considered. $\mu$ is called the diffusion potential since its gradients across the solid volume lead to the diffusion process. Note that $\mu$ depends on the type of diffusion, which can follow interstitial as well as vacancy mechanisms, according to Larché and Cahn (1973):

$J=-B\left(m, T, \sigma_{i j}\right) \nabla \mu\left(m, \sigma_{i j}\right)$

Where $B$ is the diffusing chemical species mobility, which depends upon local temperature, stress and concentration. In order to simplify the theoretical approach, the dependence of $B$ upon the chemical states will be neglected throughout the present work. In the specific case, corresponding to the conditions fulfilled in practice, when moisture diffuses in polymers, that interstitial diffusion only is assumed to occur, at a fixed temperature, $B$ satisfies the following relation, given by Larché and Cahn (1982):

$B_{i j}=D_{i j} \frac{m}{R T}$

Where $D_{i j}$ is the diffusivity. $B_{i j}$ as well as $D_{i j}$ is a second order tensor, note that $B$ and $D$ are symmetric tensors. The mass conservation equation is expressed by:

$\frac{\partial m}{\partial t}+\nabla J=0$

Assuming the stress relaxation to be associated to time constants significantly shorter than the transient part of the diffusion process, a quasi static equilibrium can be considered to take place at any moment. This additional, realistic assumption enables writing:

$\sigma_{i j, j}=0$

Relation (5) is valid for small strains only:

$\left\{\begin{array}{l}\varepsilon_{i j}^{\mathrm{tot}}=\varepsilon_{i j}^{m}+\varepsilon_{i j}^{c h} \\ \varepsilon_{i j}^{m}=S_{i j k l} \sigma_{k l}\end{array}\right.$

Where $S_{i j k l}$ denotes the compliances tensor.
The second part of the right member, $\varepsilon_{i j}^{c h}$, denotes the free strains induced by the hygroscopic expansion. The total strain is expressed as a function of displacement through:

$\varepsilon_{i j}^{\text {tot }}=\frac{\left(u_{i, j}+u_{j, i}\right)}{2}$

The diffusion potential is determined from the study of the total energy of the solid-diffusing chemical species system. In the case, investigated in the present study, of the interstitial diffusion of water molecules in polymers, the system total energy was defined by Larché and Cahn (1973) as follows:

$\mathrm{de}^{\prime}=\sigma_{i j} d \varepsilon_{i j}^{\text {total }}+\mathrm{Tds}^{\prime}+\frac{1}{\overline{V_{0}}} \sum \mu \mathrm{dm}$

Where $\bar{V}_{0}$ stands for the molar volume of the diffusing chemical species whose content is denoted by $m$, whereas $s$ represents the entropy. The symbol ' relates to the reference state of the corresponding quantity. Two Legendre transforms, involving either Helmholtz free energy density $f$, or Gibbs function $\varphi$ can be achieved:

$f^{\prime}=e^{\prime}-\mathrm{Ts}^{\prime}$

$\varphi^{\prime}=f^{\prime}-\sigma_{i j} \varepsilon_{i j}^{\text {tot }}$

Partial derivative symmetries relative to $\varphi^{\prime}$ yield the following Maxwell equation:

$\left.\left.-\frac{\partial \mu}{\partial \sigma_{i j}}\right)_{m, T}=\overline{V_{0}} \frac{\partial \varepsilon_{i j}^{\text {tot }}}{\partial m}\right)_{\sigma_{k l}, T}$

Accounting for (5) in (9), the Maxwell equation can be rewritten as:

$\left.\left.\frac{\partial \mu}{\partial \sigma_{i j}}\right)_{m, T}=-\overline{V_{0}}\left(\frac{\partial S_{i j_{k l}} \sigma_{k l}}{\partial m}\right)_{\sigma_{k l}, T}-\overline{V_{0}} \frac{\partial \varepsilon_{i j}^{c h}}{\partial m}\right)_{\sigma_{k l}, T}$

By integrating with respect to the stress tensor, one obtains:

$\mu\left(m, \sigma_{i j}\right)=\mu(m, 0)-\overline{V_{0}}\left[\left(\frac{\partial S_{i j_{k l}}}{\partial m}\right)_{\sigma_{k l}, T} \cdot \sigma_{k l} \cdot \sigma_{i j}+\beta_{i j} \sigma_{i j}\right]$

Where the coefficient of hygroscopic expansion was introduced, as follows, through its definition:

$\left.\beta_{i j}=\frac{\partial \varepsilon_{i j}^{c h}}{\partial m}\right)_{\sigma_{k l}, T}$

$\mu(m, 0)$ is the diffusing species chemical potential in the free stress state. The second order factor involved in Equation (11) expresses a possible effect of the diffusing species content on the elastic stiffness. In the following of the present work, such a dependence of the stiffness upon the moisture content will be neglected. The interested reader can nevertheless refer to Youssef et al. (2009a), where that very effect was thoroughly investigated. Moreover, amorphous polymers exhibit isotropic properties, so that $\beta_{i j}=\beta \delta_{i j}$ (where $\delta_{i j}$ stands for the Kronecker symbol). The resulting chemical potential linearly depends upon the stress tensor trace:

$\mu\left(m, \sigma_{i j}\right)=\mu(m, 0)-\overline{V_{0}} \beta \operatorname{tr} \sigma_{i j}$

With initial and appropriate boundary conditions, the Equations (1,3-6) and (11) constitute the general formulation of the coupled diffusion problem. The displacements and the content 
fields are unknown. When it is more convenient to work in terms of stresses, Equations (5) and (6) are replaced by the compatibility conditions. In this case, we get six equations for six components of stress and content. A simultaneous solving of this set of equations provides time-dependent evolutions of the stress and content fields.

\section{Application of the coupled model to the case of an isotropic solid}

\subsection{Constitutive equations}

Moisture diffusion in an isotropic polymer is considered in the present section. The material is assumed to exhibit a coefficient of moisture expansion $\beta$. Accounting for that additional assumption yields the following simplified form for (12):

$\varepsilon_{i j}^{c h}=\beta \Delta m \delta_{i j}$

Where $\Delta m=m-m_{0}$ ( $m_{0}$ standing for the initial chemical species content in the solid).

Beltrami-Mitchell compatibility equations enable expressing the studied problem in terms of stresses:

$$
(1+\nu) \sigma_{i j, k k}+\sigma_{k k, i j}+E \beta\left(\frac{1+\nu}{1-\nu} \delta_{i j} L_{, k k}+L_{, i j}\right)=0
$$

These conditions are necessary and sufficient for ensuring the solid medium continuity. They provide the displacement field from the stresses. Equations (1) to (6) become:

$$
\left\{\begin{array}{l}
J=-B \nabla \mu \\
B=\frac{D m}{R T} \\
\frac{\partial m}{\partial t}+\nabla J=0 \\
\sigma_{i j . j}=0 \\
\varepsilon_{i j}^{\text {tot }}=\frac{-\nu}{E} \operatorname{tr} \sigma_{i j} \delta_{i j}+\frac{1+\nu}{E} \sigma_{i j}+\beta \Delta m \delta_{i j}
\end{array}\right.
$$

\subsection{Determination of the diffusion law}

The diffusion law is deduced from the moisture flux, which itself is a function of the generalized chemical potential, the Expression (1) can be written as follows:

$J=-\frac{D_{m} m}{R T}\left[\frac{\partial \mu\left(m, \sigma_{i j}\right)}{\partial m} \frac{\partial m}{\partial r}+\frac{\partial \mu\left(m, \sigma_{i j}\right)}{\partial \operatorname{tr} \sigma_{i j}} \frac{\partial \operatorname{tr} \sigma_{i j}}{\partial r}\right]$

In contrast to Fick's model, Expression (17) shows that the flow does not only depend upon the local content gradient, but also on the content distribution over the entire solid. In others words, unlike uncoupled approaches, the proposed model accounts for nonlocal interactions. Moreover, moisture absorption yields internal mechanical states. According to the literature, any mechanical state may affect the so-called "free volume" of the organic matrix (Fahmy and Hurt, 1980; Neogi and Kim, 1984; Neogi et al., 1986; Neumann and Marom, 1986; Henson and Weitsman, 1986; Gillat and Broutman, 1978; Youssef et al., 2009b). Now, many authors relate the diffusion coefficients of polymers to the mechanical states through their free volume fraction (Fujita, 1961; Fahmy and Hurt, 1980; Neuman and Marom, 1985; Gueribiz et al., 2011). The resulting theoretical approach is thus often referred to as the free volume theory.
In the following of the present study, two practical examples will be investigated:

i) the case when the polymer matrix diffusivity $D_{m}$ remains independent upon the mechanical states, and ii), the case when $D_{m}$ varies as a function of the stresses.

\subsubsection{Assuming the matrix diffusivity independent upon the stress} state

In the case that the polymer diffusivity is assumed independent upon the mechanical state, one can write $D_{m}\left(\sigma_{i j}\right)=D_{m}$. The differential equation describing the diffusion law is obtained from the expression of mass conservation (3), expressed in cylindrical coordinates:

Expressing the chemical potential through Equation (13) yields:

$$
\begin{aligned}
\frac{\partial m}{\partial t}= & \frac{1}{r}\left[\frac{D_{m}}{R T} m\left(\frac{\partial \mu\left(m, \sigma_{i j}\right)}{\partial m} \frac{\partial m}{\partial r}-\overline{V_{0}} \beta \frac{\partial \operatorname{tr} \sigma_{i j}}{\partial r}\right)\right] \\
& +\frac{D_{m}}{R T} \operatorname{div}\left[m\left(\frac{\partial \mu\left(m, \sigma_{i j}\right)}{\partial m} \frac{\partial m}{\partial r}-\overline{V_{0}} \beta \frac{\partial \operatorname{tr} \sigma_{i j}}{\partial r}\right)\right]
\end{aligned}
$$

Equation (18) develops

$$
\begin{aligned}
\frac{\partial m}{\partial t}=\frac{D_{m}}{R T}\left[\left(\frac{\partial m}{\partial r}\right)^{2}\left(\frac{\partial \mu\left(m, \sigma_{i j}\right)}{\partial m}+m \frac{\partial^{2} \mu\left(m, \sigma_{i j}\right)}{\partial m^{2}}\right)\right. & \\
& \left.\left.+m \frac{\partial \mu\left(m, \sigma_{i j}\right)}{\partial m} \frac{\partial^{2} m}{\partial r^{2}}-\overline{V_{0}} \beta \frac{\partial m}{\partial r} \frac{\partial \operatorname{tr} \sigma_{i j}}{\partial r}+m \frac{\partial^{2} \operatorname{tr} \sigma_{i j}}{\partial r^{2}}\right)\right] \\
+ & \frac{1}{r} \frac{D_{m}}{R T} m\left(\frac{\partial \mu\left(m, \sigma_{i j}\right)}{\partial m} \frac{\partial m}{\partial r}-\overline{V_{0}} \beta \frac{\partial \operatorname{tr} \sigma_{i j}}{\partial r}\right)
\end{aligned}
$$

Considering:

$$
\begin{gathered}
\mu_{, 1}=\frac{\partial \mu\left(m, \sigma_{i j}\right)}{\partial m} \text { and } \mu_{, 2}=\frac{\partial^{2} \mu\left(m, \sigma_{i j}\right)}{\partial m^{2}} \text { finally we get : } \\
\begin{aligned}
\frac{\partial m}{\partial t}=\frac{D_{m}}{R T} & {\left[\mu_{, 1} \frac{1}{r} \frac{\partial m}{\partial r} m+\left(\frac{\partial m}{\partial r}\right)^{2}+m \frac{\partial^{2} m}{\partial r^{2}}\right)+\mu_{, 2} m\left(\frac{\partial m}{\partial r}\right)^{2} } \\
& \left.\left.-\overline{V_{0}} \beta \frac{\partial \operatorname{tr} \sigma_{i j}}{\partial r}\left(\frac{\partial m}{\partial r}+\frac{m}{r}\right)+m \frac{\partial^{2} \operatorname{tr} \sigma_{i j}}{\partial r^{2}}\right)\right]
\end{aligned}
\end{gathered}
$$

3.2.2. Assuming matrix diffusivity dependent upon the stress state

In this case the flux is expressed by (17) substituting (17) in the expression of mass conservation (3), one obtains:

$$
\begin{aligned}
\frac{\partial m}{\partial t}= & \frac{1}{r}\left[\frac{D_{m}\left(\sigma_{i j}\right)}{R T} m\left(\frac{\partial \mu\left(m, \sigma_{i j}\right)}{\partial m} \frac{\partial m}{\partial r}-\overline{V_{0}} \beta \frac{\partial \operatorname{tr} \sigma_{i j}}{\partial r}\right)\right] \\
& +\frac{1}{R T} \operatorname{div}\left[D_{m}\left(\sigma_{i j}\right) m\left(\frac{\partial \mu\left(m, \sigma_{i j}\right)}{\partial m} \frac{\partial m}{\partial r}-\overline{V_{0}} \beta \frac{\partial \operatorname{tr} \sigma_{i j}}{\partial r}\right)\right] \\
\frac{\partial m}{\partial t}= & \frac{1}{r}\left[\frac{D_{m}\left(\sigma_{i j}\right)}{R T} m\left(\frac{\partial \mu\left(m, \sigma_{i j}\right)}{\partial m} \frac{\partial m}{\partial r}-\overline{V_{0}} \beta \frac{\partial \operatorname{tr} \sigma_{i j}}{\partial r}\right)\right] \\
& +\frac{1}{R T} \frac{\partial D_{m}\left(\sigma_{i j}\right)}{\partial r}\left[m\left(\frac{\partial \mu\left(m, \sigma_{i j}\right)}{\partial m} \frac{\partial m}{\partial r}-\overline{V_{0}} \beta \frac{\partial \operatorname{tr} \sigma_{i j}}{\partial r}\right)\right] \\
& +\frac{D_{m}\left(\sigma_{i j}\right)}{R T} \operatorname{div}\left[m\left(\frac{\partial \mu\left(m, \sigma_{i j}\right)}{\partial m} \frac{\partial m}{\partial r}-\overline{V_{0}} \beta \frac{\partial \operatorname{tr} \sigma_{i j}}{\partial r}\right)\right]
\end{aligned}
$$


Which simplifies as follows:

$$
\begin{aligned}
\frac{\partial m}{\partial t}=\frac{1}{r}\left[\frac{D_{m}}{R T} m\left(\frac{\partial \mu\left(m, \sigma_{i j}\right)}{\partial m} \frac{\partial m}{\partial r}-\overline{V_{0}} \beta \frac{\partial \operatorname{tr} \sigma_{i j}}{\partial r}\right)\right] \\
+\frac{D_{m}}{R T}\left[\frac{\partial \mu\left(m, \sigma_{i j}\right)}{\partial m}\left(\frac{\partial m}{\partial r}\right)^{2}+m \frac{\partial^{2} \mu\left(m, \sigma_{i j}\right)}{\partial m^{2}}\left(\frac{\partial m}{\partial r}\right)^{2}\right. \\
\left.+m \frac{\partial \mu\left(m, \sigma_{i j}\right)}{\partial m} \frac{\partial^{2} m}{\partial r^{2}}-\overline{V_{0}} \beta \frac{\partial m}{\partial r} \frac{\partial \operatorname{tr} \sigma_{i j}}{\partial r}-\overline{V_{0}} \beta m \frac{\partial^{2} \operatorname{tr} \sigma_{i j}}{\partial r^{2}}\right] \\
+\frac{\partial D_{m}\left(\sigma_{i j}\right)}{\partial r} m\left(\frac{\partial \mu\left(m, \sigma_{i j}\right)}{\partial m} \frac{\partial m}{\partial r}-\overline{V_{0}} \beta \frac{\partial \operatorname{tr} \sigma_{i j}}{\partial r}\right)
\end{aligned}
$$

Finally:

$$
\begin{gathered}
\frac{\partial m}{\partial t}=\frac{1}{R T}\left[\frac{D_{m}\left(\sigma_{i j}\right)}{r}+\frac{\partial D_{m}\left(\sigma_{i j}\right)}{\partial r}\right] m \mu_{, 1} \frac{\partial m}{\partial r}-\overline{V_{0}} \beta \frac{\partial \operatorname{tr} \sigma_{i j}}{\partial r}+\frac{\partial m}{\partial t} \\
=\frac{D_{m}\left(\sigma_{i j}\right)}{R T}\left[\mu_{, 1}\left(\left(\frac{\partial m}{\partial r}\right)^{2}+m \frac{\partial^{2} m}{\partial r^{2}}\right)+\mu_{, 2} m\left(\frac{\partial m}{\partial r}\right)^{2}\right. \\
\left.\left.-\bar{V}_{0} \beta \frac{\partial m}{\partial r} \frac{\partial \operatorname{tr} \sigma_{i j}}{\partial r}+m \frac{\partial^{2} \operatorname{tr} \sigma_{i j}}{\partial r^{2}}\right)\right]
\end{gathered}
$$

In order to solve Equations (20) and (24), the global chemical potential should be estimated. This particular task requires an expression of the stress free chemical potential $\mu(m, 0)$ of the considered system diffusing chemical species/polymer matrix.

Larché and Cahn (1973) demonstrated that the chemical potential at free state $\mu(m, 0)$ may be taken as the chemical potential at reference state $\mu(m)$ for the case of an interstitial diffusion. For polymers, this potential depends on the vitrous transition temperature. Modelling the variation of chemical potential and pressure as a function of content is treated according to the nature of the couple polymer/solvent, since the sorption isotherms may show considerable discrepancies from one system to another. Details on the question of sorption isotherms can be found in Gaudichet-Maurin (2005) works. In the case that gas sorption in rubbery polymers, is considered, the relationship between the content and the pressure is often assumed to satisfy Henry's Law. Considering the moist air as a mixture of ideal gases and equating activity to content in the stress-free polymer yields:

$\mu(m)=\mu^{o}+R T \ln \left(\frac{m}{m_{0}}\right)$

Relation (25) is not appropriate for modelling strong moisture contents, since the sorption isotherm is often convex in such a situation. Convex sorption isotherms can be more accurately represented by the so-called Flory-Huggins equation:

$\mu(m, 0)=\mu^{o}+R T\left[\ln (m)+(1-m)+\chi(1-m)^{2}\right]$

Where $\chi$ is the Flory-Huggins coefficient, varying from $\chi=0.5$ to $\chi=1.5$ for rubbery or glassy polymers, respectively). Although Equation (26) works well for most polymers, other laws, more comprehensive, are often applied to the cases of either rubbery polymers or elastomers. Among these laws, Flory-HugginsRehner relation takes into account the effects of macromolecular chains cross-linking. Moreover, unlike the traditional Henry's law, it has the advantage of being valid for any value of the solubility:

$$
\begin{aligned}
\mu(m, 0)= & \mu^{o}+R T\left[\ln (m)+(1-m)+\chi(1-m)^{2}\right] \\
& +\frac{E}{3 \rho_{0}}\left[(1-m)^{1 / 3}-(1-m) / 2\right]
\end{aligned}
$$

The last part of the right side of the above written Equation (27) takes into account the elastic distortion of the polymeric chains induced by the infiltration of the diffusing species.

\section{Extension of the coupled model to composites materials}

4.1. Case of a matrix the diffusivity of which is independent of the stress state

Let us consider a composite reinforced by unidirectional, impermeable, infinitely long fibres, homogeneously embedded in a polymer matrix. The elementary unit cell of such a material can be schematized by two coaxial cylinders, one representing the fibre surrounded by the second representing the matrix (Fig. 1). The fibres have a revolution symmetry axis noted $z$.

A perfect adhesion is assumed between the fibre and the matrix. Moreover, the unit cell can be supposed to be free of macroscopic stresses. The mechanical problem posed on the REV cell can be expressed as:

\begin{tabular}{|ll}
$\operatorname{div}(\sigma)=0$ & Equilibrium in REV \\
$\sigma=L:[\varepsilon(U)-\beta \Delta m]$ & Behavior in REV \\
$\varepsilon=\frac{1}{2}\left(\nabla U+\nabla^{t} U\right)$ & Compatibility in REV \\
$\|\sigma \cdot n\|=0$ & Load transmission at interface \\
$\|U\|=0$ & Continuity of displacements at interface \\
$\langle\sigma\rangle=0$ & Macroscopic stresses (free state)
\end{tabular}

Where $\beta$ stands for the coefficients of hygroscopic expansion, whereas $L$ is the elastic stiffness tensor. These two tensors are assumed to be constant for both constituents.

Due to the cylindrical geometry of the problem, the resolution is conducted in a cylindrical coordinate system defined by the variables $(r, \theta, z)$ associated to the components of displacement vector $(w, v, u)$. Taking into account the problem geometry and the considered load, the displacement field solution of the problem (28) is written in each phase as follows:

- In the matrix

$\left\{\begin{array}{l}u(z)=A^{m} z \\ w(r)=B^{m} r+\frac{C^{m}}{r}\end{array}\right.$

- In the fibre

$$
\left\{\begin{array}{l}
u(z)=A^{f} z \\
w(r)=B^{f} r
\end{array}\right.
$$



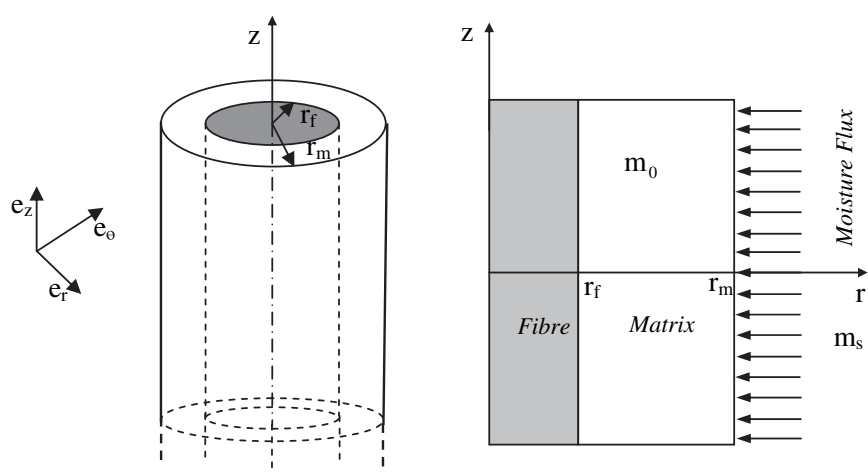

Fig. 1. Representative unit cell (REV) of unidirectional composite material.
Finally, determination of the whole displacement field depends on the determination of 5 constants, namely $A^{m}, B^{m}, C^{m}, A^{f}$ and $B^{f}$, which can be deduced from the following conditions: displacement continuity at fibre/matrix interface, continuity of radial stress at the interface fibre/matrix, boundary conditions, global stress equilibrium condition. Then, the strains and stresses are easy to deduce.

In order to solve the diffusion problem, a relation describing the moisture sorption isotherm should be chosen.

Then the differential Equation (20) is transformed to dimensionless equation using the following variables change: $\zeta=r / r_{m}$, $\tau=D_{m} t / r_{m}^{2}$.

The diffusion problem to be solved writes as follows:

$$
\left\{\begin{array}{l}
\left.\left.\frac{\partial m}{\partial \tau}=\frac{1}{R T}\left[\mu_{, 1} \frac{1}{\zeta} \frac{\partial m}{\partial \zeta} m+\left(\frac{\partial m}{\partial \zeta}\right)^{2}+m \frac{\partial^{2} m}{\partial \zeta^{2}}\right)+\mu_{, 2} m\left(\frac{\partial m}{\partial \zeta}\right)^{2}-\overline{V_{0}} \beta \frac{\partial \operatorname{tr} \sigma_{i j}}{\partial \zeta}\left(\frac{\partial m}{\partial \zeta}+\frac{m}{\zeta}\right)+m \frac{\partial^{2} \operatorname{tr} \sigma_{i j}}{\partial \zeta^{2}}\right)\right] \\
J\left(\zeta_{f}, \tau\right)=0 \quad \text { Condition at fibre/matrix interface } \\
m(1, \tau)=M_{S} \quad \text { Boundary condition } \\
m(\zeta, 0)=0 . \quad \text { Initial condition }
\end{array}\right.
$$

The components of the strain tensor are then given by the following relations:

$$
\| \begin{aligned}
& \varepsilon_{r r}=\frac{\partial w(r)}{\partial r} \\
& \varepsilon_{\theta \theta}=\frac{w(r)}{r} \\
& \varepsilon_{r \theta}=\varepsilon_{r z}=\varepsilon_{\theta z}=0 \\
& \varepsilon_{z z}=\frac{\partial u(z)}{\partial z}
\end{aligned}
$$

In the case of an isotropic material, such as the polymer matrix, the radial component $w_{m}$ of the displacement field satisfies the following equation (Jacquemin et al., 2005):

$$
r^{2} \frac{\partial^{2} w^{m}}{\partial r^{2}}+r \frac{\partial w^{m}}{\partial r}-w^{m}=\frac{k_{m} r^{2} \frac{\partial \Delta m^{m}}{\partial r}}{L_{\theta \theta}}
$$

Where: $k_{m}=L_{z \theta} \beta_{z z}+L_{\theta r} \beta_{\theta \theta}+L_{\theta \theta} \beta_{\theta \theta}$.

For isotropic material : $\beta_{z z}=\beta_{\theta \theta}=\beta$,

$$
\begin{aligned}
L_{\theta \theta} & =\frac{E(1-\nu)}{(1+\nu)(1-2 \nu)} \\
L_{\theta r} & =L_{z \theta}=\frac{E \nu}{(1+\nu)(1-2 \nu)}
\end{aligned}
$$

The solution of the differential Equation (32) is the sum of the solution of the homogeneous associated equation and a particular solution. The radial component of the displacement field in the hydrophilic matrix is obtained by considering a polynomial approximation of the transient moisture content that can easily be obtained through the classical finite difference method, as an example.
The reader should take care that the maximum moisture absorption capacity Ms at REV edges, although constant according to the traditional Fickian model, varies over time within the coupled model presented in this work. This evolution of Ms, occurring during the transient phase of the diffusion comes from the dependence of the chemical potential on the mechanical stresses states the magnitude of which change as the sorption takes place. At any time, the Ms value is obtained from equating the chemical potentials of water at the interface between the ambient fluid and the composite material.

A finite difference calculation, expressed in a cylindrical coordinate system, is applied to the system (36) through an explicit scheme, in which the moisture content at the edges varies during the diffusion process.

The material used for the simulation is a composite composed of Epoxy matrix and glass fibre, the properties of both materials are listed in Table 1:

Boundary condition corresponds to a moisture relative humidity of $100 \%$. For the value of constant $a$ of Doolittle's Equation (37), we have taken the experimentally determined value for Epoxy resin $a=0.033$. Forthe free-volume fraction $v_{0}^{f}$ in the stress-free state corresponding to the Epoxy, the value of $2.5 \%$ is taken (Neuman and Marom, 1985). The value of the molar volume of water $\overline{V_{0}}$ is taken to be $18 \mathrm{~cm}^{3} / \mathrm{mol}$.

We start the calculation process by imposing an initial value of the moisture at the edges of the REV. The bulk of the material is considered as dry at the initial stage. Thus, at start, the diffusivity is independent from the position (i.e. the depth). Besides, the initial stress state is assumed to be null throughout the studied sample. First, the diffusion problem is solved. As a result one obtains the new moisture content profile in the material, as a function of the depth. Then, the stresses are evaluated, and the mechanical states are determined. Owing to the knowledge of both the moisture content and the mechanical states, the diffusivity is calculated as a 
Table 1

Mechanical properties of glass fibre and epoxy resin N5028 (Jacquemin et al., 2005).

\begin{tabular}{lcllll}
\hline & $E(\mathrm{GPa})$ & $\nu$ & $\rho\left(\mathrm{kg} / \mathrm{m}^{3}\right)$ & $\beta$ & $M_{m}(\%)$ \\
\hline Epoxy N5028 & 4.5 & 0.3 & 1200 & 0.6 & 5.9 \\
Glass fibre & 77.5 & 0.23 & 2540 & 0 & 0 \\
\hline
\end{tabular}

function of the position as well as new boundary conditions. The next loop of the calculation process is then initiated. This numerical implementation is summarised below (Fig. 2).

Fig. 3 displays the evolution of the average moisture content in the matrix normalized to the matrix content at saturation, for different values of the matrix hygroscopic expansion varying from 0 to 0.6. The curves were plotted using Henry's Law (25) for the chemical potential of water in the polymer. According to Fig. 3, the time dependent moisture content in the matrix as well as its maximum moisture absorption capacity strongly depends on the hygroscopic expansion coefficient. Thus, one of the main factors controlling the magnitude of the hygro-mechanical coupled effects over the moisture diffusion process is the mechanical states induced by the expansion of the polymer in presence of water. The predictions of the classical Fick's law are reproduced by the coupled model in the case that the condition $\beta=0$ is assumed during the calculations. The deviation between Fick's law and the predicted results increases with $\beta$. The Fick's law is obtained as particular case when Henry's law is applied for the chemical potential of water in the polymer for $\beta=0$. Notting that the curves in Figs. 3 and 4 do not start with a neat straight line at the origin. This is due to the coupling phenomenon between moisture diffusion and internal mechanical states.

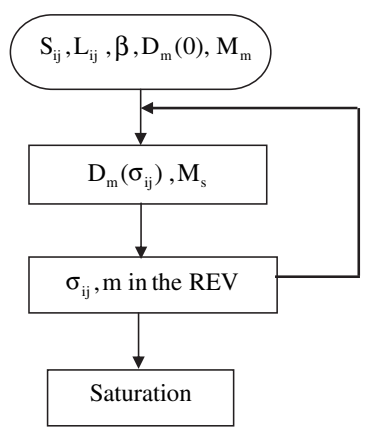

Fig. 2. Algorithm of numerical simulation.

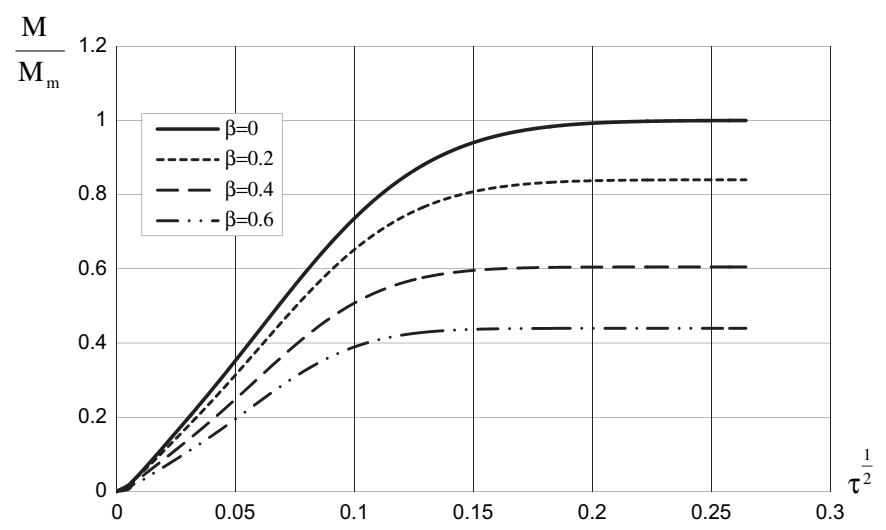

Fig. 3. Evolution of the normalized average moisture content in the composite matrix for different values of the coefficient of hygroscopic expansion $\left(v_{\mathrm{f}}=0.7\right)$.

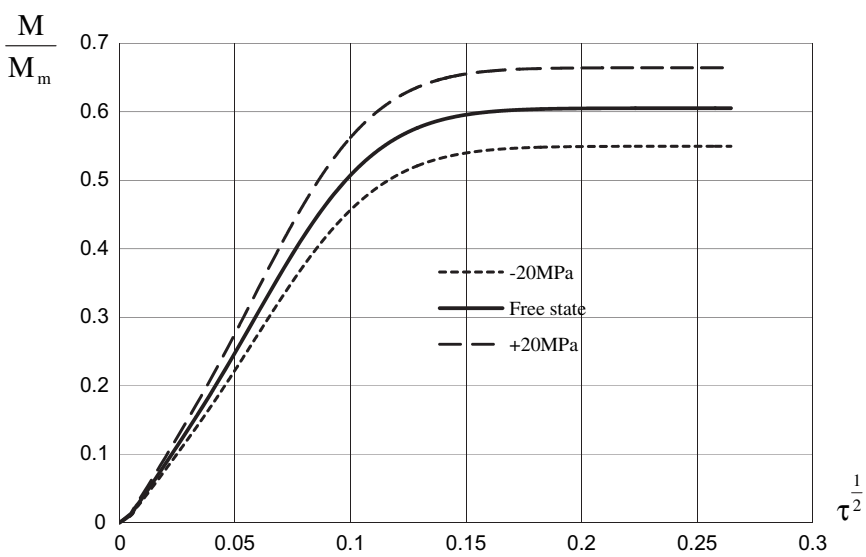

Fig. 4. Effect of an additional external mechanical load on the time-dependent profile of the normalized average moisture content in composite matrix $\left(\beta=0.4, v_{\mathrm{f}}=0.7\right)$.

Fig. 4 shows the evolution of the normalized average moisture content in the composite matrix, as a function of the external mechanical load applied to the edges of the unit cell. The sign and magnitude of the applied load affects the maximum moisture absorption capacity reached in the permanent stage of the diffusion process. However, the time necessary for reaching the permanent regime and the overall shape of the curve are unaffected by an additional external load. The predicted results are compatible with other coupled approaches such as the free volume theory: a tensile load increases the saturation level, whereas a compression decreases the maximum moisture absorption capacity (Fahmy and Hurt, 1980; Neuman and Marom, 1985, 1986; Gueribiz et al., 2011).

Fig. 5 presents the results predicted by the coupled model as a function of the theoretical expression considered for the chemical potential. According to Fig. 5, the models of Henry and FloryHuggins are very close together, although a small gap can be noticed in the permanent regime. The model of Flory-HugginsRehner is however far from both the others. This is probably due to the fact that this model takes into account the elastic distortion of the polymer chains caused by the infiltration of solvent between them, whereas neither Henry nor Flory-Huggins approach consider this effect.

Fig. 6 displays the time-dependent profiles predicted by the hygro-mechanical model for the moisture content, over the whole depth of the studied sample. The change of maximum moisture absorption capacity occurring at the edge of the specimen is clearly shown on this figure. Figs. 7 and 8 show the corresponding profiles

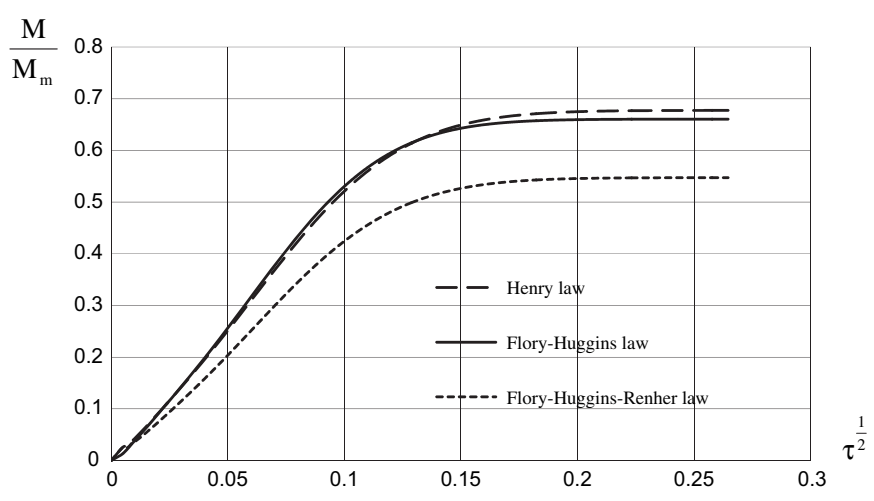

Fig. 5. Evolution of the normalized average moisture content in the organic matrix as a function of the considered chemical potential $\left(\beta=0.4, v_{\mathrm{f}}=0.7\right)$ 


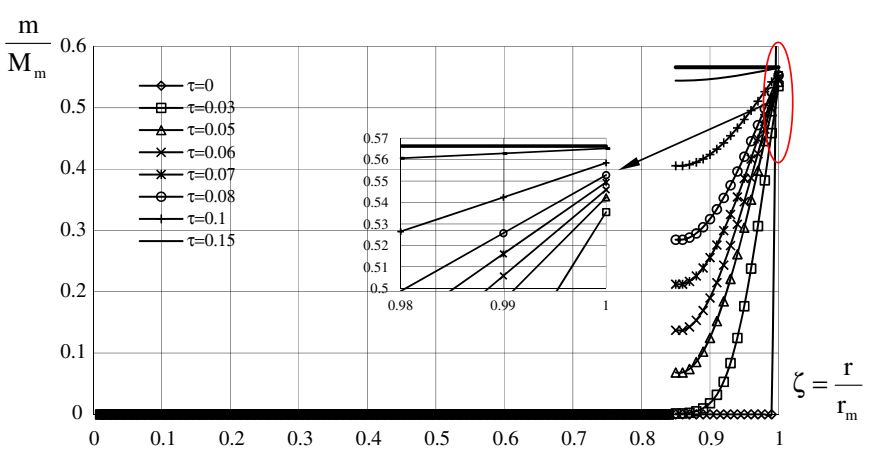

Fig. 6. In-depth time-dependent profiles predicted by the coupled model for the moisture content $\left(\beta=0.4, v_{\mathrm{f}}=0.7\right)$.

obtained for the chemical potential expressed by (13) and using Henry law for $\mu(m, 0)$ and the hydrostatic stress, respectively. A uniform value throughout the depth of the specimen is obtained for any of the studied quantities, in the permanent stage of the moisture diffusion process.

\subsection{Case of a matrix the diffusivity of which depends on the mechanical state}

In the previous section, the diffusivity of the polymer matrix was assumed to be independent from the mechanical state (thus it was remaining constant throughout the diffusion process). In this section we consider a more realistic coupling according to which the matrix diffusivity depends on the internal mechanical state. Compared to the previous case, the mechanical problem remains unchanged, as well as its solution. The difference concerns the hygroscopic problem to be solved, only:

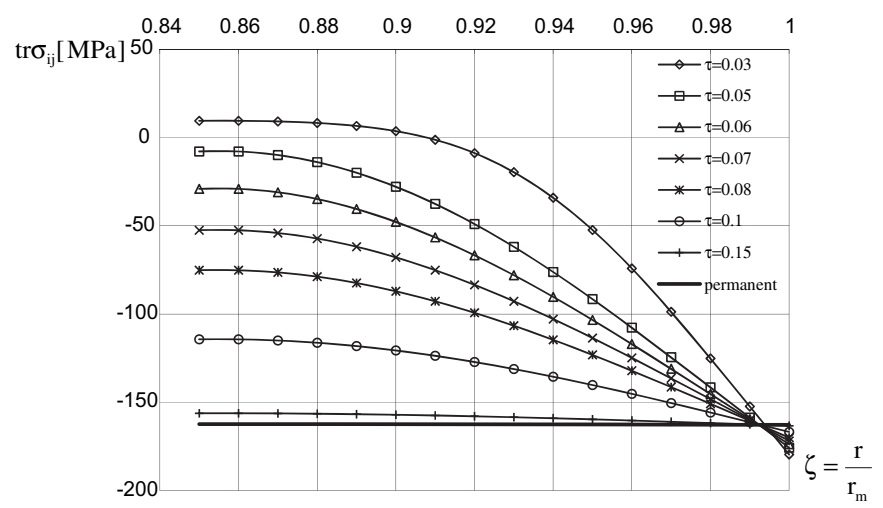

Fig. 8. In-depth time-dependent profiles predicted by the coupled model for the hydrostatic pressure experienced by the matrix $\left(\beta=0.4, v_{\mathrm{f}}=0.7\right)$.

The dependence of the matrix diffusivity on the mechanical state is evaluated on the basis of the free volume theory (Neuman and Marom, 1985):

$\left.\ln \left(\frac{D_{m}\left(\sigma_{i j}\right)}{D_{m}}\right)=a \frac{1}{v_{0}^{f}}-\frac{1}{v_{\sigma}^{f}}\right)$

Where $a$ is a proportionality constant, depending on the considered polymer. $v_{0}^{f}$ and $v_{\sigma}^{f}$ are respectively the free-volume volume fractions in the stress-free state and at a given stressed state. $D_{m}$ stands for the coefficient of moisture diffusion of the stress-free polymer, whereas $D_{m}(\sigma)$ is the corresponding coefficient in the case that the polymer experiences a given mechanical state. The relationship between $v_{0}^{f}$ and $v_{\sigma}^{f}$ is given by (Neuman and Marom, 1985):

$$
\left\{\begin{array}{l}
\left.\frac{\partial m}{\partial \tau}=\frac{1}{R T}\left[\frac{\delta_{\text {Doli }}}{\zeta}+\frac{\partial \delta_{\text {Doli }}}{\partial \zeta}\right] m \mu_{, 1}\left(\frac{\partial m}{\partial \zeta}-\overline{V_{0}} \beta \frac{\partial \operatorname{tr} \sigma_{i j}}{\partial \zeta}\right)+\frac{\delta_{\text {Doli }}}{R T}\left[\mu_{, 1}\left(\left(\frac{\partial m}{\partial \zeta}\right)^{2}+m \frac{\partial^{2} m}{\partial \zeta^{2}}\right)+\mu_{, 2} m\left(\frac{\partial m}{\partial \zeta}\right)^{2}-\overline{V_{0}} \beta \frac{\partial m}{\partial \zeta} \frac{\partial \operatorname{tr} \sigma_{i j}}{\partial \zeta}+m \frac{\partial^{2} \operatorname{tr} \sigma_{i j}}{\partial \zeta^{2}}\right)\right] \\
J\left(\zeta_{f}, \tau\right)=0 \quad \text { Condition at fibre/matrix interface } \\
m(1, \tau)=M_{S} \quad \text { Boundary condition } \\
m(\zeta, 0)=0 . \quad \text { Initial condition }
\end{array}\right.
$$

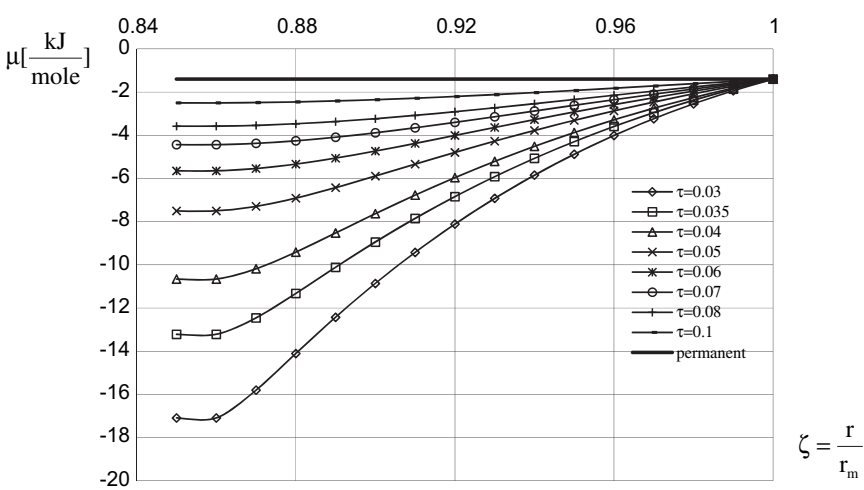

Fig. 7. In-depth time-dependent profiles predicted by the coupled model for the chemical potential $\left(\beta=0.4, v_{\mathrm{f}}=0.7\right)$.

$$
v_{\sigma}^{f}=v_{0}^{f}+\left(\frac{\Delta V}{V_{0}}\right)_{m}
$$

Accounting for (36) in relation (35), one obtains (Gueribiz et al., 2011):

$\delta_{\text {Doli }}=\frac{D_{m}^{\sigma}}{D_{m}}=\exp \left(\frac{a\left(\frac{\Delta V}{V_{0}}\right)_{m}}{v_{0}^{f}\left[v_{0}^{f}+\left(\frac{\Delta V}{V_{0}}\right)_{m}\right]}\right)$

Fig. 9 shows the time-dependent profile predicted for the average moisture content in the polymer as a function of the coefficient of hygroscopic expansion, in the case that the moisture diffusion coefficient of the matrix depends on the mechanical state. 


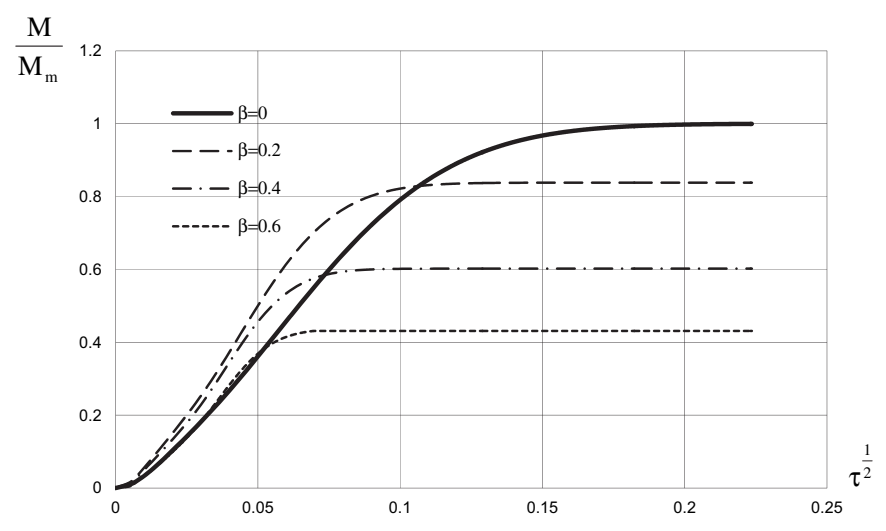

Fig. 9. Time-dependent profile predicted for the average moisture content in the polymer as a function of the coefficient of hygroscopic expansion $\left(v_{\mathrm{f}}=0.7\right)$.

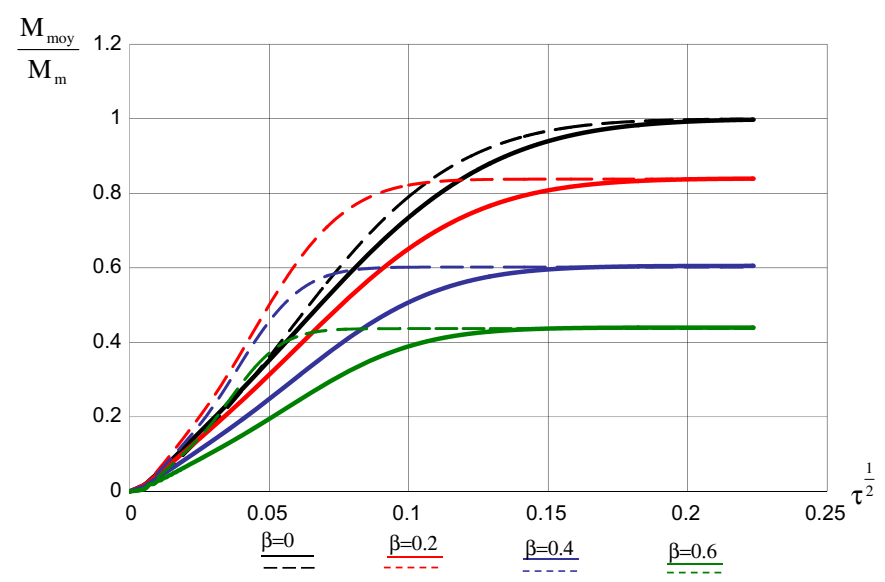

Fig. 10. Time-dependent profile predicted for the normalized average moisture content in the organic matrix (— diffusivity independent from the stresses state; ......... diffusivity depends on the stress state).

The chemical potential considered for achieving the computation corresponds to Henry's law. According to Fig. 9, the moisture content reached in the permanent stage strongly depends on the hygroscopic expansion coefficient. Low values of this coefficient are associated to high moisture content at saturation. On the contrary, strong hygroscopic expansion coefficients induce a decrease in the maximum moisture absorption capacity at the end of the transient stage of the diffusion process.

Fig. 10 displays a comparison between the predictions of the hygro-mechanical coupled model obtained in the cases that either the dependence of the diffusivity over the mechanical states is accounted for or not. It was found that the saturation level (i.e. the permanent regime) is not affected by coupling effects on the coefficient of moisture diffusion. On the contrary, accounting for an interaction between the internal mechanical state and the diffusion coefficient strongly changes the transient part of the diffusion process, leading to a shorter transient stage.

\section{Conclusion}

In this work, a realistic diffusive behaviour is investigated. A coupled modelling is proposed in which the diffusion process is considered dependent on the stress state. The formalism of such model is established based on a thermodynamic approach. The simple case of a homogeneous isotropic matrix was investigated and thereafter extended to unidirectional composite with an impermeable fibre. Due to the complexity of the featured physical processes, the coupling stress-diffusion has been treated in two stages: at first, only the coupling on the maximum moisture absorption capacity was considered, whereas the diffusivity was assumed independent of the stress state whereas, in a second time, the coupling was assumed to affect the diffusivity as well as the maximum moisture absorption capacity. The numerical computations show that the hygroscopic expansion coefficient is the parameter which has the most effect on coupling. In this study, it appears that coupling only on the content tends to increase the time required to attain the permanent regime compared to a coupling on both the maximum moisture absorption capacity and diffusivity, although that saturation levels remain unchanged. Moreover, this modelling shows that the saturation levels are lower compared to the uncoupled model and that this one overestimates the corresponding stress levels.

\section{References}

Avena, A., Bunsell, A.R., 1986. Comportement à long terme de matériaux composites en immersion sous pression hydrostatique. In: Journées Nationales sur les Composites, Paris.

Carbonell, R.G., Sarti, G.O., 1990. Coupled deformation and mass transport processes in solid polymers. Industrial \& Engineering Chemistry Research 29, 1194-1204.

Crank, J., 1975. The Mathematics of Diffusion. Clarendon Press, Oxford.

Derrien, K., Gilormini, P., 2006. Interaction between stress and diffusion in polymers. Defect and Diffusion Forum 258-260, 447-452.

Derrien, K., Gilormini, P., 2007. The effect of applied stresses on the equilibrium moisture content in polymers. ScriptaMaterialia 56, 297-299.

Durier, A.-L., Derrien, K., Gilormini, P., 2006. Effet du gonflement sur la prise d'eau de composites isotropes. In: Matériaux. Dijon.

Fahmy, A.A., Hurt, J.C., 1980. Stress dependence of water diffusion in epoxy resins. Journal of Polymer Composites 1, 77-88.

Fujita, H., 1961. Diffusion in polymer-diluent systems. Fortschritte der Hochpolymeren-Forschung (Advances in Polymer Science) 3, 1-47.

Gaudichet-Maurin, E., 2005. Caractérisation et vieillissement d'une membrane d'ultrafiltration d'eau. PhD thesis, Ecole Nationale Supérieure d'Arts et Métiers Centre de Paris.

Gillat, O., Broutman, L.J., 1978. Effect of external stress on moisture diffusion and degradation in a graphite reinforced epoxy laminate. ASTM STP 658, 61-83.

Gueribiz, D., Rahmani, M., Jacquemin, F., Fréour, S., Loucif, K., 2011. Modelling of the mechanical loading effects on the effective diffusive behavior of polymer matrix composites. Journal of Reinforced Plastics and Composites 30, 337-346.

Henson, M.C., Weitsman, Y.J., 1986. Stress Effects on Moisture Transport in an Epoxy Resin and Its Composite. In: Composite86: Recent Advances in Japan and the United States, pp. 775-783.

Jacquemin, F., Vautrin, A., 2002. A closed-form solution for the internal stresses in thick composite cylinders induced by cyclical environ- mental conditions. Composite Structure 58, 1-9.

Jacquemin, F., Fréour, S., Guillén, R., 2005. A hygro-elastic self-consistent model for fiber-reinforced composites. Journal of Reinforced Plastics and Composites 24, 485-502.

Jiming, Z., James, P.L., 1995. The effects of a water environment on anomalous absorption behavior in graphite/epoxy composites. Composites Science and Technology 53, 57-64.

Larché, F.C., Cahn, J.W., 1973. A linear theory of thermochemical equilibrium of solids under stress. ActaMetallurgica 21, 1051-1063.

Larché, F.C., Cahn, J.W., 1978. Thermochemical equilibrium of multiphase solids under stress. ActaMetallurgica 26, 1579-1589.

Larché, F.C., Cahn, J.W., 1982. the effect of self-stress on diffusion in solids. ActaMetallurgica 30, 1835-1845.

Lee, M.C., Peppas, N.A., 1993. Models of moisture transport and moisture-induced stresses in epoxy composites. Journal of Composite Materials 27, 1146-1171.

Loos, A.C., Springer, G.S., 1979. Moisture absorption of graphite-epoxy composites immersed in liquids and in humid air. Journal of Composite Materials 13, 131-147.

Loos, A.C., Springer, G.S., 1981. Environmental Effects on Composite Materials. Technomic Pub., Lancaster, PA.

Neogi, P., Kim, Myungsoo, 1984. Concentration-induced stress effects in diffusion of vapors through polymer membranes. Journal of Applied Polymers 29, 729-731.

Neogi, P., Kim, Myungsoo, Yang, Yun, 1986. diffusion in solids under strain, with emphasis on polymer membranes. AIChe Journal 32, 1146-1157.

Neuman, S., G, Marom, 1985. Stress dependence of the coefficient of moisture diffusion in composite materials. Journal of Polymer Composites 6 (1), 9-12.

Neumann, S., Marom, G., 1986. Free-volume dependent moisture diffusion under stress in composites materials. Journal of Materials Science 21, 26-30. 
Perreux, D., Choqueuse, D., Davies, P., 2002. Anomalies in moisture absorption of glass fibre reinforced epoxy tubes. Composites Part A 33, 147-154.

Shen, C.H., Springer, G.S., 1977. Environmental effects on the elastic moduli of composite materials. Journal of Composite Materials 11, 250-264

Tsai, S.W., Hahn, H.T., 1980. Introduction to Composite Materials. Technomic Publishing Co., Inc., Lancaster, Pennsylvanie.

Weitsman, Y.J., 1987. Stress assisted diffusion in elastic and viscoelastic materials. Journal of the Mechanics and Physics of Solids 35, 73-93.

Weitsman, Y., 1990. A continuum diffusion model for viscoelastic materials. Journal of Physical Chemistry 94, 961-968.

Whitney, J.M., Browning, C.E., 1978. Some anomalies associated with moisture diffusion in epoxy matrix composite materials. Advanced Composite MaterialsEnvironmental Effects, ASTM STP 658, 43-60.

Youssef, G., Fréour, S., Jacquemin, F., 2009a. Effects of moisture dependent constituents properties on the hygroscopic stresses experienced by composite structures. Mechanics of Composite Materials 45, 369-380.

Youssef, G., Jacquemin, F., Fréour, S., 2009b. Stress-dependent moisture diffusion in composite materials. Journal of Composite Materials 43, 1621-1637.

\section{Nomenclature}

$J$ : Moisture flux

$B$ : The diffusing chemical species mobility $\mu$ : The diffusion chemical potential

$m$ : Moisture content

$T$ : Temperature

$\sigma$ : Stress tensor

$\varepsilon$ : Strain tensor

$S_{i j k l}$ : The compliances tensor

$w, v, u$ : Displacements

$D_{i j}$ : The diffusivity tensor

$e$ : The system total energy

$s$ : Entropy

$\bar{V}_{0}$ : The molar volume

$f$ : Helmholtz free energy density

$\varphi$ : Gibbs function

$\beta_{i j}$ : The hygroscopic expansion tensor

$L_{i j k l}$ : The stiffness tensor

$\operatorname{tr} \sigma_{i j}$ : Trace of stress tensor

$E$ : Young's modulus

$\nu$ : Poisson's coefficien

$R$ : Ideal gas constant

$M$ : Average moisture content

$M_{m}$ : Moisture content at saturation

$M_{s}$ : The maximum moisture absorption capacity at RVE edges

$v_{0}^{f} v_{\sigma}^{f}$ : The free-volume volume fractions in the stress-free state and at given stressed state 